\title{
Discrete Random Variables Over Domains
}

\author{
M. W. Mislove ${ }^{1}$ \\ Tulane University \\ New Orleans, LA 70118
}

\begin{abstract}
In this paper we explore discrete random variables over domains. We show that these lead to a continuous endofunctor on the categories RB (domains that are retracts of bifinite domains), and FS (domains where the identity map is the directed supremum of deflations finitely separated from the identity). The significance of this result lies in the fact that there is no known category of continuous domains that is closed under the probabilistic power domain, which forms the standard approach to modeling probabilistic choice over domains. The fact that RB and FS are cartesian closed and also are closed under the discrete random variables power domain means we can now model, e.g., the untyped lambda calculus extended with a probabilistic choice operator, implemented via random variables.
\end{abstract}

\section{Introduction}

Domain theory, perhaps the most widely used method for assigning denotational meanings to programming languages, has recently seen its influence broaden to other areas of computation and mathematics. It provides a wide range of constructors for modeling data types, nondeterminism, functional programming, and several other constructs needed in semantics. It also admits a number of cartesian closed categories, the fundamental objects needed to model the lambda calculus. Even probabilistic computation admits a model in the theory, although truth to tell, this particular constructor has proven to be very difficult to unravel. Of particular interest is the question

Is there a cartesian closed category of domains that is closed under the probabilistic power domain?

There have been many attempts to resolve this, but the most we know to date is contained in [9], where it is shown that the probabilistic power domain of a finite tree is in $\mathrm{RB}$, that the probabilistic power domain of a finite reversed tree is in FS, and that RB is closed under the probabilistic power domain if the probabilistic power domain of every finite poset is in RB. But, other than finite trees, the only finite posets whose probabilistic power domain is known to be in

${ }^{1}$ This work supported by the US National Science Foundation and the US Office of Naval Research 
$\mathrm{RB}$ is the class of flat posets, whose probabilistic power domains are bounded complete (the continuous analogs of Scott domains).

We do not contribute to settling this question here, but we do provide an alternative construction - what we call the power domain of discrete random variables, which we show defines a continuous endofunctor on the category RB, as well as on FS and on CDOM, the category of coherent domains.

Objects in RB are retracts of bifinite domains, those that can be expressed as bilimits of finite posets under embedding-projection pairs. This category is cartesian closed, and it also is closed under the various power domains for nondeterminism [4]. With the addition of a mechanism to model probabilistic choice, RB provides virtually all the tools required to support semantic modeling. Furthermore, playing off results of Varacca [20,21], we show that the formation of the power domain of discrete random variables over RB yields a monad that enjoys a distributive law with respect to each of the power domain monads, and this in turn implies that each of these power domain monads lifts to a monad on the category RB that are also algebras for the discrete random variable power domain monad. In short, we can now form domain-theoretic models of computation that respect the laws of discrete random variables as well as any of the laws we choose for nondeterminism: angelic, demonic or convex choice.

The outline of the rest of the paper is as follows. In the next section we provide some background about domains and about the constructions we need. We then review briefly a construction by Varacca [21] which inspired our work, and that Varacca calls the Plotkin indexed valuations. In the following section, we investigate bag domains - domain-theoretic models for multisets, which are at the heart of our reformulation of Varacca's construction, and we then show how our approach recaptures Varacca's construction. Next we present the main result of the paper, which is to define that what we call the power domain of discrete random variables, and we show it defines a continuous endofunctor on $\mathrm{RB}, \mathrm{FS}$ and on CDOM. In the final section, we discuss further work along this line, including how to construct Varacca's other examples of indexed valuations. We also discuss the relationship between a random variable approach to modeling probabilistic computation and one based directly on probability distributions.

\subsection{Background}

We begin with some basic results about partial orders, and about domains in particular. A general reference for this material is [1] or [3].

A subset $A$ of a partially ordered set $P$ is directed if $A$ has an upper bound for each of its finite subsets. A mapping between partially ordered sets is $S$ cott continuous if it preserves the order and the suprema of those directed sets that have a supremum. A directed complete partial order (dcpo) is a partially ordered set in which each directed set has a least upper bound. A cpo is a dcpo with a least element $\perp$.

If $P$ is a partial order and $x, y \in P$, then we say $x$ is way-below $y(x \ll y)$ if whenever $A \subseteq P$ is directed and has a supremum, if $y \sqsubseteq \sqcup A$, then $x \sqsubseteq a$ for 
some $a \in A$. A poset $P$ is continuous if $\Downarrow y=\{x \in P \mid x \ll y\}$ is directed and $y=\sqcup \Downarrow y$ for each $y \in P$. A domain is a continuous dcpo.

An abstract basis is a pair $(B, \ll)$ where $\ll$ is a transitive relation on $B$ satisfying the interpolation property:

$$
F \ll x \& F \subseteq B \text { finite } \Rightarrow(\exists y \in B) F \ll y \ll x .
$$

By $F \ll x$ we mean $z \ll x \forall z \in F$. If $(B, \ll)$ is an abstract basis, then $I \subseteq B$ is a round ideal if $I$ is a $\ll-$ lower set, and $x \in I \Rightarrow(\exists y \in I) x \ll y$. The round-ideal completion of an abstract basis $(B, \ll)$ is the family of round ideals, ordered by inclusion. This forms a domain, where $I \ll J$ iff $(\exists x \ll y \in B) I \subseteq \Downarrow x \subseteq \Downarrow y \subseteq$ $J$. In fact, every domain $P$ is isomorphic to the round-ideal completion of an abstract basis, namely $P$ is isomorphic to the round ideal completion of $(P, \ll)$ under the mapping sending a point $x$ to $\Downarrow x$, whose inverse is the mapping that sends a round ideal to its supremum.

One of the fundamental results about dcpos is that the family of Scott continuous maps between two dcpos is another dcpo in the pointwise order. Since it's easy to show that the finite product of a family of continuous posets is another such, and the one-point poset is a terminal object, a central question is which categories of dcpos and Scott continuous maps are cartesian closed. This is true of DCPO, but not of DOM, the category of domains. Still, there are several full subcategories of DOM that are cartesian closed. Among the notable such categories are:

BCD Bounded complete domains, in which every non-empty subset has a greatest lower bound.

RB Domains which are retracts of bifinite domains, themselves bilimits of families of finite posets under embedding-projection maps; these are pairs of Scott continuous mappings $e: P \rightarrow Q$ and $p: Q \rightarrow P$ satisfying $p \circ e=1_{P}$ and $e \circ p \leq 1_{Q}$.

FS Domains $D$ satisfying the property that the identity map is the directed supremum of selfmaps $f: D \rightarrow D$ each finitely separated from the identity: i.e., for each $f$ there is a finite subset $M_{f} \subseteq D$ with the property that, for each $x \in D$, there is some $m \in M_{f}$ with $f(x) \leq m \leq x$.

Actually, BCD is a full subcategory of RB, which in turn is a full subcategory of FS, and FS is a maximal ccc of domains. An interesting (some might say frustrating) open question is whether RB and FS are equal. The objects in all of these categories are coherent, ${ }^{2}$ but the category CDOM of coherent domains and Scott continuous maps is not a ccc.

We also recall some facts about categories. A monad on a category $\mathrm{A}$ is a triple $(T, \mu, \eta)$ where $T: \mathrm{A} \rightarrow \mathrm{A}$ is an endofunctor, and $\mu: T^{2} \longrightarrow T$ and $\eta:{ }_{1} \mathrm{~A} \longrightarrow T$ are natural transformations satisfying the laws:

$$
\mu \circ T \mu=\mu \circ \mu_{T} \quad \text { and } \quad \mu \circ \eta_{T}=T=\mu \circ T \eta
$$

\footnotetext{
${ }^{2}$ A domain is coherent if its Lawson topology is compact; cf. [1]
} 
If $(T, \mu, \eta)$ is a monad, then a $T$-algebra is a pair $(a, h)$, where $a \in \mathrm{A}$ and $h: T a \rightarrow a$ is an A-morphism satisfying $h \circ \eta_{a}=1_{a}$ and $h \circ T h=h \circ \mu_{a}$. For example, each of a power domains, $\mathcal{P}_{L}(P), \mathcal{P}_{U}(P)$ and $\mathcal{P}_{C}(P)$ define monads on DCPO (cf. [4]), whose algebras are ordered semilattices; another example is the probabilistic power domain $\mathbb{V}(P)$ (cf. [7]), whose algebras satisfy equations that characterize the probability measures over $P$.

One goal of domain theory is to provide a setting in which all of the constructors needed to model a given programming language can be combined. If the aim is to model both nondeterminism and probabilistic choice, then one needs to combine the appropriate nondeterminism monad with the probabilistic power domain monad, in order that the laws of each constructor be preserved in the resulting model. This is the function of a distributive law, which is a natural transformation $d: S T \longrightarrow T S$ between monads $S$ and $T$ on A satisfying several identities - cf. [2]. The significance of distributive laws is a theorem of Beck [2], which says there is a distributive law of $S$ over $T$ if and only if $T$ lifts to a monad on the category of $S$-algebras. Unfortunately, it was shown by Plotkin and Varacca [20] that there is no distributive law of $\mathbb{V}$ over $\mathcal{P}_{X}$, or of $\mathcal{P}_{X}$ over $\mathbb{V}$ for any of the nondeterminism monads $\mathcal{P}_{X}$. This led to the work we report on next.

\section{Indexed Valuations}

We now recall some of the work of Varacca [21] that was motivated by problems associated with trying to model both nondeterminism and probabilistic choice. Once it was shown that there is no distributive law between $\mathbb{V}$ and any of the nondeterminism monads, Varacca realized that weakening one of the laws of probabilistic choice could result in a monad that enjoys a distributive law with respect to a monad for nondeterminism. For $0<p<1$ and $A$ a domain element, the law in question is

$$
p A+(1-p) A=A
$$

which he weakened in three ways:

$$
\begin{gathered}
p A+(1-p) A \sqsubseteq A \\
p A+(1-p) A \sqsubseteq A \\
p A+(1-p) A \text { and } A \text { unrelated by order. }
\end{gathered}
$$

He called the monad he constructed satisfying (2) the Hoare indexed valuations, the one satisfying (3) the Smyth indexed valuations and the one satisfying (4), a non-relation, the Plotkin indexed valuations. We exploit this last constructionthe so-called Plotkin indexed valuations over a domain - in defining our power domain of discrete random variables. 


\subsection{Plotkin Indexed Valuations}

An indexed valuation over the poset $P$ is a tuple $\left(r_{i}, p_{i}\right)_{i \in I}$ where $I$ is an index set, ${ }^{3}$ each $r_{i} \geq 0$ is a non-negative real number and $p_{i} \in P$ for each $i \in I$. Two indexed valuations satisfy $\left(r_{i}, p_{i}\right)_{I} \simeq_{1}\left(s_{j}, q_{j}\right)_{J}$ if $|I|=|J|$ and there is a permutation $\phi \in S(|I|)^{4}$ with $r_{\phi(i)}=s_{i}$ and $p_{\phi(i)}=q_{i}$ for each $i$. If we let $I^{\prime}=\left\{i \in I \mid r_{i} \neq 0\right\}$ and similarly for $J$, then $\left(r_{i}, p_{i}\right)_{I} \simeq_{2}\left(s_{j}, q_{j}\right)_{J}$ if $\left(r_{i}, p_{i}\right)_{I^{\prime}} \simeq_{1}\left(s_{j}, q_{j}\right)_{J^{\prime}}$, and we let $\simeq$ denote the equivalence relation on indexed valuations generated by $\simeq_{2}$. For an indexed valuation $\left(r_{i}, p_{i}\right)_{I}$, we let $\left\langle r_{i}, p_{i}\right\rangle_{I}$ denote the equivalence class modulo $\simeq$.

Next, let $\overline{\mathbb{R}_{>0}}$ denote the extended non-negative real numbers, with the usual order. Then for a domain $P$, Varacca defines a relation $\ll_{P}$ on the family $\left\{\left\langle r_{i}, p_{i}\right\rangle_{I} \mid r_{i} \in \overline{\mathbb{R}_{\geq 0}} \& p_{i} \in P\right\}$ of indexed valuations over $P$ by

$$
\begin{aligned}
\left\langle r_{i}, p_{i}\right\rangle_{I} \ll_{P}\left\langle s_{j}, q_{j}\right\rangle_{J} \quad \text { iff } \quad\left(\left|I^{\prime}\right|=\left|J^{\prime}\right|\right)\left(\exists \phi \in S\left(\left|I^{\prime}\right|\right)\right) \\
r_{i}<s_{\phi(i)} \& p_{i} \ll_{P} q_{\phi(i)}\left(\forall i \in I^{\prime}\right) .^{5}
\end{aligned}
$$

Note that we can "add" indexed valuations $\left\langle r_{i}, p_{i}\right\rangle_{I}$ and $\left\langle s_{j}, q_{j}\right\rangle_{J}$ by simply concatenating the tuples and taking the equivalence class of the resulting $I \dot{U} J$ tuple. This forms a continuous operation on indexed valuations that is commutative, by construction. We also can let $\overline{\mathbb{R}_{+}}$act on $\left\langle r_{i}, p_{i}\right\rangle_{I}$ by $r \cdot\left\langle r_{i}, p_{i}\right\rangle_{I}=$ $\left\langle r \cdot r_{i}, p_{i}\right\rangle_{I}$. Varacca's main result for the family of Plotkin indexed valuations is:

Theorem 1 (Varacca [20]).

- If $P$ is a continuous poset, then the family of Plotkin indexed valuations ordered by $\ll_{P}$ as defined in (5) is an abstract basis. The family $I V_{P}(P)$, the round ideal completion of the Plotkin indexed valuations, satisfies the following family of equations:

$$
\begin{array}{ll}
\text { (1) } A \oplus B=B \oplus A & \text { (2) } A \oplus(B \oplus C)=(A \oplus B) \oplus C \\
\text { (3) } A \oplus \underline{0}=A & \text { (4) } 0 A=\underline{0} \\
\text { (5) } 1 A=A & \text { (6) } p(A \oplus B)=p A \oplus p B \\
\text { (7) } p(q A)=(p q) A & \text { where } p, q \in \mathbb{R}_{+} \text {and } A, B, C \in I V_{P}(P) \text {. }
\end{array}
$$

- The Plotkin indexed valuation defines the object level of a functor which is monadic over DOM, and each of the power domain monads satisfies a distributive law with respect to the Plotkin indexed valuations monad.

A corollary of this result is that the composition $\mathcal{P}_{P} \circ I V_{P}$ defines a monad on CDOM, the category of coherent domains, whose algebras satisfy the laws listed in Theorem 1 and the laws of the Plotkin power domain:

$$
\text { (i) } X+Y=Y+X \text { (ii) } X+X=X \text { (iii) } X+(Y+Z)=(X+Y)+Z
$$

In other words, $\mathcal{P}_{P}\left(I V_{P}(P)\right)$ is the initial domain semilattice algebra over $P$ that also satisfies the laws listed in Theorem 1.

\footnotetext{
${ }^{3}$ For our discussion, we can assume $I$ is always finite.

${ }^{4} S(n)$ denotes the permutation group on $n$.

${ }^{5}$ Note that $r<s$ iff $r \ll s$ for $r, s \in \mathbb{R}$.
} 


\section{Bag domains}

In this section we develop some results that are fundamental for our main construction. The details of these results are contained in [15]. The construction of bag domains - domains whose elements are bags or multisets from an underlying domain, originated in the work of Vickers [22], and also have been considered by Johnstone $[5,6]$. Those works were inspired by work in database theory, and the goals of their work was to capture the abstract categorical nature of the construction. Here we present results along the same line, but we provide a more direct construction that allows us to analyze the internal structure of the objects more closely. It also allows us to capture the constructions of Varacca [20] more concretely. We begin with a simple result about posets:

Definition 1. Let $P$ be a poset and let $n \in \mathbb{N}$. For $\phi \in S(n)$, define a mapping $\phi: P^{n} \rightarrow P^{n}$ by $\phi(d)_{i}=d_{\phi^{-1}(i)}$. Then $\phi$ permutes the components of $d$ according to $\phi$ 's permutation of the indices $i=1, \ldots, n$. Next, define a preorder $\bigsqcup_{n}$ on $P^{n}$ by

$$
d \sqsubseteq_{n} e \quad \text { iff } \quad(\exists \phi \in S(n)) \phi(d) \leq e \quad \text { iff } \quad d_{\phi^{-1}(i)} \leq e_{i}(\forall i=1 \ldots, n) .
$$

Finally, we define the equivalence relation $\equiv$ on $P^{n}$ by

$$
\equiv=\sqsubseteq_{n} \cap \sqsupseteq_{n},
$$

and we note that $\left(P^{n} / \equiv, \sqsubseteq_{n}\right)^{6}$ is a partial order. We denote by $[d]$ the image of $d \in P^{n}$ in $P^{n} / \equiv$.

Lemma 1. Let $P$ be a poset and let $n \in \mathbb{N}$. Then the following are equivalent:

1. $d \sqsubseteq_{n} e$,

2. $(\exists \phi \in S(n))(\forall i=1, \ldots, n) d_{i} \leq e_{\phi(i)}$, for $i=1, \ldots, n$.

3. $\uparrow\{\phi(d) \mid \phi \in S(n)\} \supseteq \uparrow\{\phi(e) \mid \phi \in S(n)\}$.

Proof. For (i) implies (ii), we note that, if $\phi \in S(n)$ satisfies $d_{\phi^{-1}(i)} \leq e_{i}$, then $d_{i} \leq e_{\phi(i)}$, for each $i=1, \ldots, n$, so (ii) holds. Next, (ii) implies $\phi^{-1}(e) \in \uparrow d$, and then $\psi(e) \in \uparrow\{(\phi(d) \mid \phi \in S(n)\}$ for each $\psi \in S(n)$ by composing permutations, from which (iii) follows. Finally, (iii) implies (i) is clear.

We also need a classic result due to M.-E. Rudin [10]

Lemma 2 (Rudin). Let $P$ be a poset and let $\left\{\uparrow F_{i} \mid i \in I\right\}$ be a filter basis of non-empty, finitely-generated upper sets. Then there is a directed subset $A \subseteq$ $\cup_{i} F_{i}$ with $A \cap F_{i} \neq \emptyset$ for all $i \in I$.

Next, let $P$ be a dcpo and let $n>0$. We can apply the lemma above to derive the following:

$\overline{{ }^{6} \text { We use } \sqsubseteq_{n}}$ to denote the $\sqsubseteq / \equiv$, the partial order on $P^{n} / \equiv$ induced by the pre-order defined above. 
Proposition 1. Let $P$ be a dcpo, and let $n>0$.

- If $A \subseteq P^{n} / \equiv$ is directed, then there is a directed subset $B \subseteq \bigcup_{[a] \in A}\{\phi(a) \mid$ $\phi \in S(n)\}$ satisfying

$$
\uparrow\{\phi(\sqcup B) \mid \phi \in S(n)\}=\bigcap_{a \in A} \uparrow\{\phi(a) \mid \phi \in S(n)\} \quad \text { and } \quad[\sqcup B]=\sqcup A .
$$

- In particular, $\left(P^{n} / \equiv, \sqsubseteq\right)$ is a dcpo, and the mapping $x \mapsto[x]: P^{n} \rightarrow P^{n} / \equiv$ is Scott continuous.

Proof. If $A \subseteq P^{n} / \equiv$ is directed, then Lemma 1 implies that $\left\{\cup_{\phi \in S(n)} \uparrow \phi(a) \mid\right.$ $[a] \in A\}$ is a filter basis of finitely generated upper sets, and so by Lemma 2 there is a directed set $B \subseteq \bigcup_{[a] \in A}\{\phi(a) \mid \phi \in S(n)\}$ with $B \cap\{\phi(a) \mid \phi \in S(n)\} \neq \emptyset$ for each $[a] \in A$. Since $B \subseteq P^{n}$ is directed, we have $x=\sqcup B$ exists. If $[a] \in A$, then $B \cap\{\phi(a) \mid \phi \in S(n)\} \neq \emptyset$ means there is some $\phi \in S(n)$ with $\phi(a) \in B$, so $\phi(a) \leq x$ by Lemma 1 . Hence $a \sqsubseteq x$ for each $[a] \in A$, so $[x]$ is an upper bound for $A$.

We also note that, since $\sqcup B=x$,

$$
\bigcap_{b \in B} \uparrow\{\phi(b) \mid \phi \in S(n)\}=\uparrow\{\phi(x) \mid \phi \in S(n)\} .
$$

Indeed, the right hand side is clearly contained in the left hand side since $b \leq x$ for all $b \in B$. On the other hand, if $y$ is in the left hand side, then $b \sqsubseteq y$ for each $b \in B$. Now, since $S(n)$ is finite, there is some $\phi \in S(n)$ and some cofinal subset $B^{\prime} \subseteq B$ with $\phi(b) \leq y$ for each $b \in B^{\prime}$. But then $\sqcup B^{\prime}=\sqcup B$, and so $\sqcup\left\{\phi(b) \mid b \in B^{\prime}\right\}=\phi(x)$, from which we conclude that $\phi(x) \leq y$. Thus $y$ is in the right hand side, so the sets are equal.

Now, if $y \in P^{n}$ satisfies $a \sqsubseteq y$ for each $[a] \in A$, then since $B \subseteq \bigcup_{[a] \in A}\{\phi(a) \mid$ $\phi \in S(n)\}$, it follows that $b \sqsubseteq y$ for each $b \in B$. Then $y \in \bigcap_{b \in B} \uparrow\{\phi(b) \mid \phi \in$ $S(n)\}=\uparrow\{\phi(x) \mid \phi \in S(n)\}$, and so $x \sqsubseteq y$. Thus $[x]=\sqcup A$ in the order $\sqsubseteq_{n}$. This also shows the $\bigcap_{[a] \in A} \uparrow\{\phi(a) \mid \phi \in S(n)\}=\uparrow\{\phi(x) \mid \phi \in S(n)\}$

It is clear now that $P^{n} / \equiv$ is a dcpo, and the argument we just gave shows that directed sets $B \subseteq P^{n}$ satisfy $[\sqcup B]=\sqcup_{b \in B}[b]$.

Proposition 2. Let $P$ be a domain and let $n \in \mathbb{N}$. Then

1. $\left(P^{n} / \equiv, \sqsubseteq_{n}\right)$ is a domain. Moreover, $\left[d_{i}\right]_{n} \ll\left[e_{i}\right]_{n}$ iff $(\exists \phi \in S(n)) \phi\left(\left(d_{i}\right)_{n}\right) \ll\left(e_{i}\right)_{n}$.

2. If $P$ is $\mathrm{RB}$ or $\mathrm{FS}$, then so is $P^{n} / \equiv$.

3. If $P$ is coherent, then so is $P^{n} / \equiv$.

Proof. $P^{n} / \equiv$ is a domain: Proposition 1 shows that $\left(P^{n} / \equiv, \sqsubseteq_{n}\right)$ is directed complete and that the quotient map is Scott continuous. To characterize the way-below relation on $P^{n} / \equiv$, let $x, y \in P^{n}$ with $x \ll y$. Then $x_{i} \ll y_{i}$ for each $i=1, \ldots, n$, and it follows that $\phi(x) \ll \phi(y)$ for each $\phi \in S(n)$. If $A \subseteq P^{n} / \equiv$ is directed and $[y] \sqsubseteq_{n} \sqcup A$, then there is some $\phi \in S(n)$ with $\phi(y) \leq z$, where 
$[z]=\sqcup A$. Then Proposition 1 shows there is a directed set $B \subseteq \cup_{[a] \in A} \uparrow\{\phi(a) \mid$ $\phi \in S(n)\}$ with $\sqcup B \equiv z$. Hence, there is some $\psi \in S(n)$ with $\psi(y) \leq \sqcup B$. Since $\psi(x) \ll \psi(y)$, it follows that there is some $b \in B$ with $\psi(x) \leq b$, so $[x] \sqsubseteq_{n}[b]$. Hence $[x] \ll[y]$ in $D^{n} / \equiv$.

We have just shown that $x \ll y$ in $P^{n}$ implies that $[x] \ll[y]$ in $P^{n} / \equiv$. Since $D^{n}$ is a domain, $\downarrow y$ is directed with $y=\sqcup \downarrow y$, and so the same is true for $\downarrow[y] \in D^{n} / \equiv$. Thus $P^{n} / \equiv$ is a domain.

$P^{n} / \equiv$ is RB if $P$ is: Now suppose the $P$ is in RB. Then, by Theorem 4.1 of [8] there is a directed family $f_{k}: P \rightarrow P$ of Scott continuous maps with $1_{P}=\sqcup_{k} f_{k}$ and $f_{k}(P)$ finite for each $k \in K$. Then the mappings $\left(f_{k}\right)^{n}: P^{n} \rightarrow P^{n}$ also form such a family, showing $P^{n}$ is in RB.

Next, given $k \in K, x \in P^{n}$ and $\phi \in S(n)$, we have $\phi\left(f_{k}^{n}(x)\right)=f_{k}^{n}(\phi(x))$ since $f_{k}^{n}$ is $f_{k}$ acting on each component of $x$. It follows that there is an induced map $\left[f_{k}^{n}\right]: P^{n} / \equiv \rightarrow P^{n} / \equiv$ satisfying $\left[f_{k}^{n}\right]([x])=\left[f_{k}^{n}(x)\right]$, and this map is continuous since [ ] is a quotient map. Finally, $\left[f_{k}^{n}\right]\left(P^{n} / \equiv\right)$ is finite since $f_{k}^{n}\left(P^{n}\right)$ is finite, and that $\sqcup_{k}\left[f_{k}^{n}\right]=1_{P^{n} \equiv}$ follows from $\sqcup_{k} f_{k}^{n}=1_{P^{n}}$. Thus, $P^{n} / \equiv$ is RB is $P$ is.

$P^{n} / \equiv$ is FS if $P$ is: Continuing, the domain $P$ is FS if there is a directed family of selfmaps $f_{k}: P \rightarrow P$ satisfying $\sqcup_{k} f_{k}=1_{P}$ and for each $k \in K$, there is some finite $M_{k} \subseteq P$ with $f_{k}(x) \leq m_{x} \leq x$ for some $m_{x} \in M_{k}$, for each $x \in P$. The remainder is similar to the case of RB.

$P^{n} / \equiv$ is coherent if $P$ is: Last, we consider coherent domains. Recall a domain is coherent if the Lawson topology is compact, where the Lawson topology has for a basis the family of sets $\{U \backslash \uparrow F \mid F \subseteq P$ finite \& $U$ Scott open $\}$. Now, if $x \in P^{n}$, then $\{\phi(x) \mid \phi \in S(n)\}$ is finite, and so if $F \subseteq P^{n} / \equiv$ is finite, then $[\uparrow F]^{-1}=\cup_{[x] \in F} \uparrow\{\phi(x) \mid \phi \in S(n)\}$ is finitely generated. It follows that [ ]: $P^{n} \rightarrow P^{n} / \equiv$ is Lawson continuous, so if $P$ is coherent, then so are $P^{n}$ and $P^{n} / \equiv$.

Definition 2. For a domain $P$, we let $\mathcal{B}_{n}(P)=P^{n} / \equiv$ denote the domain of $n$-bags over $P$. We also let $\mathcal{B}(P)=\oplus_{n} \mathcal{B}_{n}(P)$ denote the coalesced sum $^{7}$ of the $\mathcal{B}_{n}(P)$, where we identify $P^{0} \equiv \underline{0}$.

\section{Theorem 2.}

1. $\mathcal{B}_{n}$ defines a continuous endofunctor on the categories DCPO, DOM, CDOM, $\mathrm{RB}$ and $\mathrm{FS}$ for each $n \in \mathbb{N}$.

2. $\mathcal{B}$ defines a continuous endofunctor on DCPO and DOM, as well as on $\mathrm{CDOM}_{\perp}, \mathrm{RB}_{\perp}$ and $\mathrm{FS}_{\perp} .8$

3. In fact, $\mathcal{B}$ defines the monad of commutative monoids over each of the categories listed in 2.

Proof. $\mathcal{B}_{n}$ is defined as a composition of constructors that define continuous endofunctors on each of the categories, so it is continuous.

\footnotetext{
7 The coalesced sum of cpos takes their disjoint union and identifies their least elements.

${ }^{8}$ If $A$ is a category of dcpos, the $A_{\perp}$ denotes the subcategory of cpos in $A$ and maps that preserve least elements.
} 
For $\mathcal{B}$, we must add the countable coalesced sum operator, which it is easy to show leaves each of the indicated categories invariant.

The fact that $\mathcal{B}(P)$ is a monoid follows by defining $\left\langle p_{i}\right\rangle_{m} \oplus\left\langle q_{j}\right\rangle_{n}=\left\langle r_{k}\right\rangle_{m+n}$, where $r_{k}=p_{k}$ for $k \leq m$ and $r_{k}=q_{m+k}$ otherwise, with $P^{0} \equiv \underline{0}$ as the identity.

Example 1. If $P=\{\perp, a, b, \top\}$ is the four-element lattice with $a$ and $b$ incomparable, then $P^{2} / \equiv$ is not in BCD: the pair $[a, \perp],[b \perp]$ has $[a, b]$ and $[T, \perp]$ as minimal upper bounds. ${ }^{9}$

\subsection{Reconstructing $I V_{P}(P)$}

We now use our results on bag domains to reconstruct Varacca's Plotkin indexed valuations. The facts we need are contained in the following.

Definition 3. We define the family of real $n$-bags over a domain $P$ by $\mathcal{B}_{n}^{\mathbb{R}}(P)=$ $\mathcal{B}_{n}\left(\overline{\mathbb{R}_{\geq 0}} \times P\right) /\left\{\left[r_{i}, d_{i}\right]_{n} \mid(\exists i) r_{i}=0\right\}$. We also define $\mathcal{B}^{\mathbb{R}}(P)=\oplus_{n} \mathcal{B}_{n}^{\mathbb{R}}(P)$.

Theorem 3. $\mathcal{B}_{n}^{\mathbb{R}}$ and $\mathcal{B}^{\mathbb{R}}$ define continuous endofunctors on $\mathrm{CDOM}, \mathrm{RB}$ and on FS. Moreover, $\mathcal{B}^{\mathbb{R}}$ defines a monad on each of these categories whose algebras satisfy the laws (1) - (7) of Theorem 1.

Proof. $\mathcal{B}_{n}^{\mathbb{R}}(P)$ is the quotient of $\mathcal{B}_{n}\left(\overline{\mathbb{R}_{\geq 0}} \times P\right)$ modulo a Scott-closed subset, which shows $\mathcal{B}_{n}^{\mathbb{R}}(P)$ is in CDOM, RB or FS if $P$ is. The definition of $\mathcal{B}^{\mathbb{R}}(P)$ implies $\underline{0}$ is the least element, which implies $\mathcal{B}^{\mathbb{R}}$ leaves these categories invariant.

Given $f: P \rightarrow Q$, we define $\mathcal{B}_{n}^{\mathbb{R}}(f)\left(\left[r_{i}, d_{i}\right]_{n}\right)=\left[r_{i}, f\left(d_{i}\right)\right]_{n}$, and then $\mathcal{B}^{\mathbb{R}}(f)=$ $\oplus_{n} \mathcal{B}_{n}^{\mathbb{R}}(f)$. Clearly $\mathbb{R}_{+}$acts on $\mathcal{B}_{n}^{\mathbb{R}}(P)$, and the last part follows from the fact that these Scott-closed subsets are invariant with respect to this action.

Theorem 4. For a domain $P, I V_{P}(P) \simeq \mathcal{B}^{\mathbb{R}}(P)$.

Proof. This follows by noting that the mapping $\left\langle r_{i}, p_{i}\right\rangle_{n} \mapsto\left[r_{i}, p_{i}\right]_{n}$ and $\underline{0} \mapsto \underline{0}$ defines a bijection that takes $\ll_{P}$ on $I V_{P}(P)$ to $\ll$ on $\mathcal{B}^{\mathbb{R}}(P)$.

Corollary 1. Each of the power domain monads $\mathcal{P}_{X}$ lifts to a monad on $\mathcal{B}^{\mathbb{R}_{-}}$ algebras.

Proof. Varacca showed that there is a distributive law of $I V_{P}$ over $\mathcal{P}_{X}$ in [20], and this implies that $\mathcal{P}_{X}$ lifts to a monad on the class of $\mathcal{B}^{\mathbb{R}}$-algebras by Beck's Theorem [2] and by Theorem 4 . In fact, we can easily recover the distributive law that Varacca obtains in [21]: the distributive law $d: \mathcal{B}^{\mathbb{R}} \mathcal{P}_{X} \longrightarrow \mathcal{P}_{X} \mathcal{B}^{\mathbb{R}}$ can be defined as $d_{P}\left(\left[r_{i}, X_{i}\right]_{n}\right)=\left\langle\left[r_{i}, x_{i}\right]_{n} \mid x_{i} \in X_{i} \in \mathcal{P}_{X}(P)\right\rangle$. The result follows.

\footnotetext{
${ }^{9}$ Thanks to one of the anonymous referees for this example.
} 


\section{Random variables over domains}

We now show how to construct the power domain of discrete random variables over a domain. Recall that a random variable is a function $f:(X, \mu) \rightarrow(Y, \Sigma)$ where $(X, \mu)$ is a probability space, $(Y, \Sigma)$ is a measure space, and $f$ is a measurable function, which means $f^{-1}(A)$ is measurable in $X$ for every $A \in \Sigma$, the specified $\sigma$-algebra of subsets of $Y$. Most often random variables take their values in $\mathbb{R}$, equipped with the usual Borel $\sigma$-algebra. For us, $X$ will be a countable, discrete space, and $Y$ will be a domain, where $\Sigma$ will be the Borel $\sigma$-algebra generated by the Scott-open subsets.

Given a random variable $f: X \rightarrow Y$, the usual approach is to "push the probability measure $\mu$ forward" onto $Y$ by defining $f \cdot \mu(A)=\mu\left(f^{-1}(A)\right)$ for each measurable subset $A$ of $Y$. But this defeats one of the attractions of random variables: namely, that there may be several points $x \in X$ which $f$ takes to the same value $y \in Y$. This is 'attractive' because it means that the random variable $f$ makes distinctions that the probability measure $f \cdot \mu$ does not, and we would like to exploit this fact. Varacca makes exactly this point in his work [20,21], a point he justifies by showing how to distinguish the random variable $f$ from the probability measure $f \cdot \mu$ operationally. We return to this point later. For the moment, we define our power domain of random variables.

Definition 4. For a domain $P$, we define the power domain of discrete random variables over $P$ to be the subdomain

$$
\mathbb{R} \mathbb{V}(P)=\bigcup_{n}\left\{\left[r_{i} d_{i}\right]_{n} \mid n \geq 1 \& \sum_{i} r_{i} \leq 1\right\} \cup\{\underline{0}\} \subseteq \mathcal{B}^{\mathbb{R}}(P) .
$$

Remark 1. We can think of a discrete random variable over $P$ as a formal sum $\sum_{i \leq n} r_{i} \delta_{x_{i}}$ where some of the $x_{i}$ can be repeated. But, the order from $\mathcal{B}^{\mathbb{R}}(P)$ distinguishes, for example, $\frac{1}{2} \delta_{x} \oplus \frac{1}{2} \delta_{x}$ from $\delta_{x}$, while these two would be identified as probability measures.

We now come to our main result.

Theorem 5. $\mathbb{R V}$ defines a continuous endofunctor on CDOM, RB and FS.

Proof. $\mathbb{R} \mathbb{V}$ is obtained by restricting $\mathcal{B}^{\mathbb{R}}$ in the "real components" to ones whose sum is at most 1 , which defines a Scott-closed subset of $\mathcal{B}^{\mathbb{R}}(P)$. Hence $\mathbb{R} \mathbb{V}(P)$ is in each of the indicated categories if $P$ is. Since continuous maps $f: P \rightarrow Q$ are extended to $\mathcal{B}^{\mathbb{R}}(P)$ by $\mathcal{B}^{\mathbb{R}}(f)\left[r_{i}, p_{i}\right]=\left[r_{i}, f\left(p_{i}\right)\right]$ and since the elements in $\mathbb{R} \mathbb{V}(P)$ are those in $\mathcal{B}^{\mathbb{R}}(P)$ whose real components sum to at most 1 ; it follows that $\mathcal{B}^{\mathbb{R}}(f)$ preserves this property in $\mathcal{B}^{\mathbb{R}}(Q)$. The result follows.

Corollary 2. Each of the power domain monads $\mathcal{P}_{X}$ lifts to a monad on $\mathbb{R} \mathbb{V}$ algebras.

Proof. The distributive law given in the proof of Corollary 1 clearly restricts to one for $\mathbb{R V}$. 
This corollary means we can solve domain equations such as $P \simeq \mathcal{P}_{X} \circ \mathbb{R} \mathbb{V}(P)$ for each of the power domain monads $\mathcal{P}_{X}$. The resulting domain $P$ will be a $\mathcal{P}_{X^{-}}$ algebra and simultaneously a $\mathbb{R} \mathbb{V}$-algebra.

One might also ask about the relationship between our construction and the traditional probabilistic power domain over a domain. The following provides the answer.

Theorem 6. If $P$ is a domain, then there is an epimorphism Flat: $\mathbb{R V}(P) \rightarrow$ $\mathbb{V}(P)$, the domain of valuations over $P$.

Proof. The mapping Flat is defined by Flat $\left(\left[r_{i}, d_{i}\right]_{n}\right)=\sum_{i \leq n} r_{i} \delta_{d_{i}}$, where in $\mathbb{V}(P)$, summands with the same support are identified. This is easily seen to define a continuous map. It is an epimorphism of domains because the simple valuations are dense [7], and clearly they are the range of Flat.

\section{Summary and Future Work}

We have presented a power domain for discrete random variables, and shown that it enjoys a distributive law with respect to each of the power domain monads. Moreover, our construction defines a continuous endofunctor on the cartesian closed categories RB and FS, as well as on the category CDOM. This is where our results on bag domains have their payoff, since trying to devise these last results using abstract bases would be much more difficult. Varacca actually presents three separate constructions, as indicated in Section 2. Our methods can be adopted to recapture each of them; a discussion of the Hoare indexed valuations from our approach is presented in [15]. Since no similar result is known to hold for the probabilistic power domain, our construction provides an alternative for modeling probabilistic choice on domains.

One issue we haven't discussed is what sort of operational intuition there is for random variables. Again, we rely on Varacca, who showed in [21] that, for a simple state-based language supporting nondeterminism and probabilistic choice, probabilistic schedulers could distinguish distinct programs in his model. This is similar to refusal testing in CSP: one tests a process at each place where a probabilistic choice is made. In the more traditional approach using probabilistic bisimulation, such as in [16], one tests processes at the end of their computation, not at each stage. This provides a viable, albeit more complicated method of assigning behaviors to programs.

Another issue not discussed here is whether one can bring Shannon's information theory into the picture [18]. This is based on bringing entropy into play; there are some very interesting results about domains and entropy in Martin's recent work [12], a line we plan to explore. A particularly appealing issue here is defining an order on random variables over a domain relative to which entropy forms a measurement. If Martin's work is any indication, this will probably be a fairly difficult issue to resolve.

Acknowledgment We wish to thank the anonymous referees for many valuable suggestions. 


\section{References}

1. Abramsky, S. and A. Jung, "Doman Theory," in: Handbook of Logic in Computer Science, S. Abramsky and D. M. Gabbay and T. S. E. Maibaum, editors, Clarendon Press, 1994, pp. 1-168.

2. Beck, J., Distributive laws, in: Semian on Triples and Categorical Homology Theory, 1969, pp. 119-140.

3. Gierz, G., K. H. Hofmann, K. Keimel, J. Lawson, M. Mislove and D. Scott, "Continuous Lattices and Domains," Cambridge University Press, 2003.

4. Hennessy, M. and G. D. Plotkin, Full abstraction for a simple parallel programming language, Lecture Notes in Computer Science 74 (1979), pp. 108-120.

5. Johnstone, P. T., Partial products, bag domains and hyperlocal toposes, LMS Lecture Notes Series 77 (1992), pp. 315-339.

6. Johnstone, P. T., Variations on a bagdomain theme, Theoretical Computer Science 136 (1994), pp. 3-20.

7. Jones, C., "Probabilistic Nondeterminism," PhD Dissertation, University of Edinburgh, Scotland, 1989.

8. Jung, A., "Cartesian Closed Categories of Domains," CWI Tracts 66 (1989), Centrum voor Wiskunde en Informatica, Amsterdam.

9. Jung, A. and R. Tix, The problematic probabilistic power domain, Electronic Notes in Theoretical Computer Science 13 (1999), http://www.elsevier.com/locate/ entcs/volume13.html

10. Lawson, J. D., The upper interval topology, property $\mathcal{M}$ and compactness, Electronic Notes in Theoretical Computer Science 13 (1998), http://www.elsevier. com/locate/entcs/volume13.html.

11. Lowe, G., "Probabilities and Priorities in Timed CSP," DPhil Thesis, Oxford University, 1993.

12. Martin, K. Entropy as a fixed point, Proceedings of ICALP 2004, LNCS 3142, 2004.

13. Mislove, M. Algebraic posets, algebraic cpos and models of concurrency, Proceedings of the Oxford Symposium on Topology, G. M. Reed, A. W. Ros-coe and R. Wachter, editors, Oxford University Press, $75-111$.

14. Mislove, M. Nondeterminism and probabilistic choice: Obeying the laws, Lecture Notes in Computer Science 1877 (2000), pp. 350-364.

15. Mislove, M. Monoids over domains, submitted to MSCS, 2005.

16. Mislove, M., J. Ouaknine and J. B. Worrell, Axioms for probability and nondeterminism, Proceedings of EXPRESS 2003, Electronic Notes in Theoretical Computer Science 91(3), Elsevier.

17. Morgan, C., et al, Refinement-oriented probability for CSP, Technical Report PRGTR-12-94, Oxford University Computing Laboratory, 1994.

18. Shannon, C., A mathematical theory of information, Bell Systems Technical Journal 27 (1948), pp. 379-423 \& 623-656.

19. Tix, R., "Continuous D-Cones: Convexity and Powerdomain Constructions," PhD Thesis, Technische Universität Darmstadt, 1999.

20. Varacca, D., The powerdomain of indexed valuations, Proceedings 17th IEEE Symposium on Logic in Computer Science (LICS 2002), IEEE Press, 2002.

21. Varacca, D., "Probability, Nondeterminism and Concurrency: Two Denotational Models for Probabilistic Computation," PhD Dissertation, Aarhus University, Aarhus, Denmark, 2003.

22. Vickers, S. Geometric theories and databases, LMS Lecture Notes Series 77 (1992), pp. 288-314. 